\title{
Blood cancer health disparities in the United States Hispanic population
}

\author{
Alfonso E. Bencomo-Alvarez, Andres J. Rubio, Mayra A. Gonzalez, \\ and Anna M. Eiring
}

Department of Molecular and Translational Medicine, Paul L. Foster School of Medicine, Texas Tech University Health Sciences Center El Paso, El Paso, Texas 79905, USA

\begin{abstract}
Cancer is a challenging, multifaceted disease that involves a combination of biological and nonbiological factors. Aside from COVID-19, cancer is the second leading cause of death in the United States and the first among Hispanic Americans. The Hispanic population is the largest minority group in the United States, which is rapidly growing in size. Unfortunately, U.S. Hispanics and other minority groups experience many different health disparities, resulting in poor survival outcomes and a reduced quality of life. Factors such as genomic mutations, lower socioeconomic status, lack of education, reduced access to health care, comorbidities, and environmental factors all contribute to these health-care inequalities. In the context of blood cancer health disparities, Hispanic patients are often diagnosed at a younger age and have worse outcomes compared with non-Hispanic individuals. In this commentary, we highlight the existing knowledge about cancer health disparities in the Hispanic population, with a focus on chronic and acute leukemia. In our experience at the U.S./Mexican border, analysis of several different blood cancers demonstrated that younger Hispanic patients with acute lymphoid or myeloid leukemia have higher incidence rates and worse prognoses. A combined approach, involving improved health-care access and better knowledge of the underlying factors, will allow for more timely diagnoses and the development of intervention strategies aimed at reducing or eliminating the disparities.
\end{abstract}

Corresponding author: anna.eiring@ttuhsc.edu

(c) 2021 Bencomo-Alvarez et al. This article is distributed under the terms of the Creative Commons Attribution-

NonCommercial License, which permits reuse and redistribution, except for commercial purposes, provided that the original author and source are credited.

Published by Cold Spring Harbor Laboratory Press

doi:10.1101/mcs.a005967

\section{INTRODUCTION}

Health disparities are defined as variations in the incidence, prevalence, mortality, and burden of diseases among certain population groups (National Academies of Sciences et al. 2017). Health disparities in the United States are disproportionately observed in minority groups for many different diseases, resulting in higher death rates and an increased cost of care (National Academies of Sciences et al. 2017). In 2018, for example, health care disparities in the United States accounted for $\$ 93$ billion in excess health-care costs and $\$ 42$ billion in lost productivity, for a total of $\$ 135$ billion in annual losses (Turner 2018). Moreover, the annual per capita spending for the treatment of minority health disparities could reach $\$ 230$ billion by 2050 ( $\$ 150$ billion in medical care expenses and $\$ 80$ billion for lost productivity) (Turner 2018). The underlying factors contributing to health disparities in minority groups are not fully understood but are thought to encompass a mix of biological and nonbiological features.

A minority group is defined as a subgroup of the population with unique social, religious, ethnic, racial, or other characteristics that differ from the majority. This includes any group 
that is subjected to oppression or discrimination by those in more powerful positions, regardless of whether the group is a numerical minority (Perkins and Wiley 2014). The term Hispanic/Latino refers to people of Cuban, Puerto Rican, Mexican, South/Central American, or other Spanish-speaking cultures, regardless of race (NAACCR 2011). However, most studies documenting health disparities in Hispanic groups lump all patients into one single category, despite having heterogeneity in race, incidence, and outcomes (Hindorff et al. 2018; Rebbeck et al. 2018; Gurdasani et al. 2019). Hispanics are often grouped based on the social definitions of race and ethnicity and not necessarily on genomic ancestry or biological traits. According to the American Cancer Society, the Hispanic population represents $18 \%$ of the total population with nearly 60 million people, and that number is expected to reach 106 million Hispanic Americans by 2050 (ACS 2018). Hispanic ethnicity is often associated with reduced socioeconomic status (SES), as the median household income for Hispanics in the United States was reported to be 30\% less than the income of non-Hispanic whites (NHWs) (DaNavas-Walt and Proctor 2015).

Cancer is a leading public health problem worldwide that affects every population across the globe. Although cancer is the second cause of death in the U.S. population, it is the first cause of death among Hispanic Americans (ACS 2018). Despite being the first cause of death, Hispanic cancer patients are reported to have lower mortality rates compared with non-Hispanics for many different diseases (DaNavas-Walt and Proctor 2015; McDonald and Paulozzi 2019). This is known as the "Hispanic paradox," because a lower SES is usually associated with worse overall health and higher mortality rates (Sorlie et al. 1995; McDonald and Paulozzi 2019). Although recent progress has been made in cancer therapy, there are still significant disparities when racial and ethnic minority groups are considered, especially in medically underserved populations or those with lower SES (Philips et al. 2013; Paulozzi et al. 2020; Springfield et al. 2020). Cancer health disparities describe the differences in cancer outcomes among various population groups and are measured in terms of cancer incidence, prevalence, stage at diagnosis, morbidity, and mortality, as well as variations in screening, survivorship, and quality of life (Patel et al. 2020). Despite the "Hispanic paradox," health disparities have been reported in the Hispanic population for several different cancers, including gastric cancer (Rajabi et al. 2012; Florea et al. 2019), ovarian cancer (Shokar et al. 2019), cervical cancer (Carrillo et al. 2021), and several types of blood cancers (ACS 2020; Bencomo-Alvarez et al. 2020). Additionally, minority groups tend to have lower rates of preventive screening measures (Florea et al. 2019; Shokar et al. 2019), higher numbers of comorbidities (de Heer et al. 2013), and higher rates of complications associated with therapy (Yanez et al. 2016). Here, we summarize the burden of cancers in the U.S. Hispanic population, with a focus on chronic and acute leukemia. A better understanding of cancer health disparities in the U.S. Hispanic population will identify diseases that require timely and aggressive treatment approaches and will pave the way toward a personalized medicine approach in minorities.

\section{BLOOD CANCER HEALTH DISPARITIES IN HISPANICS}

It was estimated that 1,898,160 new cancer cases and 608,570 cancer deaths would occur in the United States in 2021 (Siegel et al. 2021). Of these new cancer diagnoses, 186,400 patients are expected to have some form of blood cancer, including myeloma (34,920 cases), lymphoma ( 90,390 cases), or leukemia (61,090 cases), and approximately 57,750 of those are expected to die from their disease (Siegel et al. 2021). Leukemia is a type of blood cancer arising from neoplastic transformation of hematopoietic stem cells in the bone marrow. Leukemia is classified into four main groups based on the type of cell affected (myeloid or lymphoid), and their rate of growth, with slower-growing leukemia termed as "chronic" 
and faster-growing leukemia known as "acute." Here, we will focus on four specific types of leukemia, including acute lymphoblastic leukemia (ALL), acute myeloid leukemia (AML), chronic lymphoblastic leukemia (CLL), and chronic myeloid leukemia (CML). In 2017, there were 434,982 people living with or in remission from leukemia in the United States (SEER 2020), and it was calculated that there will be 61,090 new diagnoses and 23,660 deaths from leukemia in the United States in 2021 alone (Siegel et al. 2021).

Leukemia is commonly diagnosed in the Hispanic population, ranking seventh on the list of cancer-related deaths (ACS 2018). Among children (0-14 yr) and adolescents (15-19 yr), Hispanic leukemia patients have higher incidence rates compared with all other racial and ethnic groups in the United States (Fajardo-Gutiérrez et al. 2007; Barrington-Trimis et al. 2015; Deshpande et al. 2015; Kahn et al. 2016a; Gramatges et al. 2017). The incidence rate of leukemia in Hispanic children is 62.6 per million, compared with 52.2 per million in $\mathrm{NHW}$ children. This clear disparity in the incidence of leukemia in Hispanic children is also reflected in the 5-yr survival rate in ALL (90\% for Hispanic; $95 \%$ for non-Hispanic) and AML (68\% for Hispanic; 74\% for non-Hispanic) (ACS 2018). Less is known about disparities in adults with blood cancers. We will outline what is currently known about incidence and survival for Hispanic adults with different types of leukemia and offer suggestions to improve the outcomes in this high-risk patient population.

\section{ACUTE LEUKEMIAS}

Acute leukemia is the result of accelerated and uncontrolled growth in early hematopoietic stem and progenitor cells, caused by mutational events that prevent the maturation of lymphoid or myeloid precursors (Appelbaum 2020). The rapid expansion of immature cell populations of lymphoid or myeloid origin results in the development of $A L L$ or $A M L$, respectively. In 2021, it is expected that 5690 new cases of ALL and 20,240 new cases of AML will be diagnosed, with higher incidence rates in males than in females for both groups (ALL: 1.9/ 100,000 for males, 1.5/100,000 for females; AML: 5.2/100,000 for males, 3.5/100,000 for females), resulting in 1580 deaths from ALL and 11,400 deaths from AML (ACS 2020). Leukemic expansion disrupts bone marrow homeostasis, leading to loss of the production of normal red blood cells, white blood cells, and platelets. When left untreated, these aggressive types of leukemia can become fatal within months of diagnosis. However, with a timely diagnosis and proper therapy, life can be prolonged and the quality of life improved (Appelbaum 2020).

\section{Acute Lymphoblastic Leukemia}

ALL is a relatively rare disease but remains the most common cancer in children (roughly onequarter of childhood cancers) and is the leading cause of death by disease in this age group (Shoag et al. 2020). Unfortunately, the incidence of ALL has continued to climb significantly in all racial and ethnic groups over the last 20 years. The incidence of ALL in the United States is much higher in the Hispanic population compared with other races and ethnicities (Pollyea et al. 2014; Swords et al. 2016; Shoag et al. 2020). Notably, the rate of increase in ALL incidence in the Hispanic population is over twofold faster compared with non-Hispanic groups (Shoag et al. 2020). Cancer health disparities for children with ALL have been extensively documented in many investigations over several decades (Lim et al. 2014; Wang et al. 2015; Kirtane and Lee 2017; Siegel et al. 2017; Shoag et al. 2020). In adults, Hispanics diagnosed with ALL are diagnosed at a younger age compared with NHWs, demonstrating a higher incidence rate and more dismal prognoses. Specifically, Hispanic ALL patients demonstrate significantly worse overall survival (OS) and progression-free survival rates, as well as higher mortality in age-adjusted analyses (Patel et al. 2012; Pulte et al. 2013; Kahn et al. 
2016b; Jain et al. 2017; Krakora et al. 2020; Shoag et al. 2020). Our own research in Texas has confirmed these findings, showing that Hispanic ALL patients are diagnosed at a significantly younger age, have higher incidence rates (RR, 1.92; 95\% Cl, 1.79-2.08; $P<0.001)$, and worse OS compared with NHWs. Additionally, patients diagnosed in El Paso, Texas, defined as Texas Health Service Region 10 (HSR10) near the U.S./Mexican border, were shown to have worse OS compared with patients diagnosed in other areas of Texas (BencomoAlvarez et al. 2020). Interestingly, this was true for both Hispanic and NHW ALL patients.

Biological factors have been associated with reduced survival in Hispanic ALL patients. Lim et al. identified five genomic loci associated with worse outcomes in Hispanic ALL patients, including ARID5B (10q21.2), IKZF1 (7p12.2), CEBPE (14q11.2), CDKN2A (9p21.3), and BMI1-PIP4K2A (10p12.31-12.2). Through genome-wide association studies (GWASs), these mutations were associated with a genetic predisposition to develop ALL and were more common in Hispanic compared with NHW patients (Lim et al. 2014). The presence of single-nucleotide polymorphisms (SNPs) in genes such as CYP1A1 and GATA3, as well as gene rearrangements of CRLF2, were also associated with higher incidence of ALL in the Hispanic population (Pollyea et al. 2014).

The evidence that ALL has a higher incidence and worse OS in the Hispanic population is indisputable. In spite of our knowledge of factors associated with this disease, we have yet to translate these findings into improved outcomes for Hispanic ALL patients. A coordinated effort will be required to translate genetic or biological markers associated with Hispanic ALL patients into improved diagnosis and care.

\section{Acute Myeloid Leukemia}

AML is the most frequent acute leukemia diagnosed in adults, representing $33 \%$ of all leukemias. Importantly, $>60 \%$ of newly diagnosed AML patients are $60 \mathrm{yr}$ of age or older. Patients with AML experience a wide range of symptoms. This is due to the immense heterogeneity of the disease, resulting from diverse gene mutations and chromosomal abnormalities, making the clinical management of AML very challenging (Stauder et al. 2020). The most treatable form of $A M L$ is acute promyelocytic leukemia (APL), a highly curable disease by treatment with arsenic trioxide and all-trans retinoic acid (Swords et al. 2016). Despite being curable, it has been widely documented that APL has higher incidence rates in the Hispanic population, in addition to presenting at younger ages compared with NHWs. Data for AML from 2010-2014 indicated that NHWs have a higher age-adjusted incidence of disease $(4.3 / 100,000)$ compared with African Americans $(3.5 / 100,000)$, Asian Americans (3.4/100,000), and Hispanics (3.6/100,000) (Kirtane and Lee 2017), with the exception of APL, which has higher incidence rates in Hispanics (Pollyea et al. 2014; Swords et al. 2016). However, Hispanic patients have worse OS at 5 and $10 \mathrm{yr}$ when compared with the $\mathrm{NHW}$ population, and this difference is more evident in younger age groups (Patel et al. 2012; Kahn et al. 2016b).

There are clear disparities among Hispanic and Black AML populations. Despite being younger at diagnosis and having a higher prevalence of favorable cytogenetic features, Black and Hispanic AML patients have increased mortality compared with NHWs (Patel et al. 2013; Patel et al. 2015; Kirtane and Lee 2017; Bhatnagar et al. 2020). Our own observations suggest once again that not only Hispanic ethnicity, but also proximity to the U.S./Mexican border, associates with worse outcomes in AML patients (RR, 1.18; $95 \% \mathrm{Cl}$, 1.04-1.33). In contrast with our observations in ALL, Hispanic but not NHW AML patients residing near the U.S./Mexican border demonstrated a worse OS compared with patients living in nonborder areas, suggesting that regional location also plays a role in AML outcomes in the Hispanic population (Bencomo-Alvarez et al. 2020). Although outcomes and 5-yr OS for AML have been improving over the last 30 years, the improvement has not been equally 
COLD SPRING HARBOR Molecular Case Studies
Blood cancer health disparities in the United States distributed among different populations. In NHW patients with AML (excluding APL), ageadjusted survival increased from $12 \%$ in 1991-1996 to $16 \%$ in 2003-2008. Similar statistics were demonstrated for both Black (from 8\% to 12\%) and Asian (from 11\% to 17\%) Americans. In contrast, survival for Hispanic AML patients did not change much in the same timeframe, with survival only improving from $13 \%$ to $14 \%$, which was not statistically significant (Kirtane and Lee 2017). The disparity is even more evident in younger populations (15-54 yr), in which there was no change in survival at all (Kirtane and Lee 2017).

Hispanic patients with AML are often observed to have favorable genetic markers (e.g., mutations in ASXL1 and TET2, as well as the $t(8 ; 21)$ and $t(15 ; 17)$ genomic translocations). However, the presence of these favorable markers does not translate into improved clinical outcomes (Patel et al. 2012, 2013, 2015; Darbinyan et al. 2017; Kirtane and Lee 2017). Additional biological factors contributing to this health disparity include mutations in CEBPA, RUNX1, GATA2, AML1-ETO, U2AF1, and KIT, which were observed at a higher prevalence in Hispanic versus NHWs. In contrast, mutations in FLT3-ITD, DNMT3A, NPM1, and IDH2 were underrepresented in Hispanics (Gomez-Arteaga et al. 2020). However, mutated FLT3 was recently shown to have a negative impact on outcomes in Mexican children (Molina Garay et al. 2020).

\section{CHRONIC LEUKEMIAS}

One of the main characteristics of chronic leukemias is the clonal expansion of partially mature cells, which may prevent them from fulfilling their immunological functions; they occur more frequently in the elderly. Nearly half of chronic leukemia patients are diagnosed in the absence of any symptoms, often through routine health exams, because chronic leukemia takes longer to develop symptoms compared with acute leukemia (Hallek 2019; Jabbour and Kantarjian 2020). CLL is a well-defined lymphoid neoplasm characterized by the proliferation and accumulation of mature $B$ cells in the blood, bone marrow, and lymphoid tissues. CLL is caused by the clonal production of B cells from a leukemic stem cell (LSC) with very heterogeneous biological and clinical presentation (Hallek 2019; Julio et al. 2020). CML, in contrast, is a rare disease caused by the clonal expansion of leukemic stem and progenitor cells, because of the presence of a balanced $t(9 ; 22)$ chromosomal translocation known as the Philadelphia chromosome. The $t(9 ; 22)$ genomic translocation results in production of the BCR-ABL1 fusion oncogenic tyrosine kinase, which is targetable through treatment with tyrosine kinase inhibitors (TKIs) (Mughal et al. 2016; Jabbour and Kantarjian 2020). In 2021, it is expected that there will be 21,520 new cases of CLL and 9110 new cases of CML in the United States (Siegel et al. 2021), with higher incidence rates in males than in females for both cancers (CLL: 6.8/100,000 for males, 3.5/100,000 for females; CML: 2.4/100,000 for males, 1.4/100,000 for females). Of those, 4320 deaths for CLL and 1220 deaths for CML are expected to occur (Siegel et al. 2021).

\section{Chronic Lymphoblastic Leukemia}

CLL is the most common type of leukemia in the United States, accounting for $35 \%$ of all new leukemia cases (ACS 2020). The disease typically occurs in elderly patients, with a median age at diagnosis of $72 \mathrm{yr}$ (Hallek 2019). The clinical course of CLL is highly variable and affects males more than females (Hallek 2019). As CLL incidence rates increase with age, the prevalence and mortality of CLL is expected to increase in the coming decades, because of demographic changes and increased life expectancy. In addition, an increase in the proportion of younger patients with early-stage CLL has been observed, likely because of more frequent blood tests (Hallek 2019). 
CLL treatment has greatly improved in recent years, thanks to the introduction of therapies involving chemotherapy combined with molecularly targeted therapies. For example, a combination of the murine and human chimeric monoclonal antibody known as rituximab (which targets CD20-positive B cells), in addition to first line of chemotherapy with fludarabine and cyclophosphamide, improves progression-free survival in $20 \%(\mathrm{HR}=0.56[95 \% \mathrm{Cl} 0.46-$ $0.69], P<0.0001)$ and $\mathrm{OS}$ in $4 \%(\mathrm{HR}=0.67$ [95\% $\mathrm{Cl} 0.48-0.92] ; P=0.01)$ of $\mathrm{CLL}$ patients compared with patients receiving chemotherapy alone (Hallek et al. 2010). However, these therapies were developed in clinical trials in which minority groups were grossly underrepresented and therefore may not translate to all populations of CLL patients (Hallek et al. 2010).

Research has shown that the NHW population has a higher incidence of CLL compared with Hispanics (Nabhan et al. 2014). Therefore, biological differences in disease presentation could render minority groups less responsive to therapies that are confirmed only in nonHispanic groups (Di Bernardo et al. 2008; Slager et al. 2010; Hernandez-Caballero et al. 2015; Bencomo-Alvarez et al. 2020). In a large population-based study, Nabhan et al. (2014) demonstrated that CLL is indeed more prevalent in NHWs, with $>85 \%$ of all cases reported for those of $>15 \mathrm{yr}$, followed by Black (6.8\%), Hispanic (4.9\%), and Asian (2.8\%) populations. Our own observations have confirmed this finding for NHW versus Hispanic CLL patients diagnosed near the U.S./Mexican border (Bencomo-Alvarez et al. 2020). Despite higher incidence rates in NHW CLL patients, minority populations were shown to present with CLL at significantly younger ages compared with NHWs. Furthermore, Hispanics were shown to have a lower SES and higher levels of poverty, whereas a higher SES was observed in non-Hispanics (Bencomo-Alvarez et al. 2020). Finally, it was demonstrated that survival of patients with CLL has improved in non-Hispanics, Hispanics, and Blacks over time, but no improvement was observed in the Asian population (Nabhan et al. 2014).

In a GWAS, Di Bernardo et al. (2008) reported six genetic susceptibility variants associated with risk of developing CLL in the Caucasian population in the United Kingdom (rs872071, rs11083846, rs7176508, rs13397985, rs735665, rs17483466). Similar polymorphisms were associated with CLL in Caucasian patients in the United States (Slager et al. 2010). In the Hispanic population of Mexico, in contrast, only the variant rs872071 was frequently observed (Hernandez-Caballero et al. 2015), possibly explaining the lower incidence of CLL observed in the Hispanic population (Bencomo-Alvarez et al. 2020). Future studies should determine whether CLL therapies identified in NHW populations are appropriate and efficacious in minority populations.

\section{Chronic Myeloid Leukemia}

CML is a myeloproliferative neoplasm characterized by the presence of the BCR-ABL1 fusion oncoprotein and is highly treatable with TKls targeting BCR-ABL1 (O'Hare et al. 2012; Minciacchi et al. 2021). TKls have greatly improved survival for CML patients across the globe, generating a life expectancy approaching that of the general population (ACS 2020; Jabbour and Kantarjian 2020). This disease is more common in adults of $>60 \mathrm{yr}$ of age and is more common in males $(2.4 / 100,000)$ compared with females $(1.4 / 100,000)$. Using data from the Surveillance Epidemiology and End Results (SEER) database, CML was shown to have a lower incidence rate in the Hispanic versus NHW population (Lee et al. 2009; Bencomo-Alvarez et al. 2020; Jabbour and Kantarjian 2020), similar to observations in CLL.

Results from several recent publications show that, in $\mathrm{CML}$, there is no significant difference in survival and/or diagnosis between Hispanics and NHWs (Lee et al. 2009; Höglund et al. 2015; Zhao et al. 2018; Utuama et al. 2019). However, these investigations only compared survival rates and no other social or environmental aspects that could reveal novel disparities. One study by Sarmad et al. (2017) demonstrated that reduced survival for CML minority patients was associated with a lack of health insurance that prevents timely and 
aggressive treatment in Black and Hispanic populations. In our own observations at the U.S./ Mexican border, NHWs have a higher incidence of CML compared with Hispanics. However, Hispanic CML patients were diagnosed at a significantly younger age, and those residing near the U.S./Mexican border had a worse OS compared with Hispanic patients living in nonborder regions. This was not due to differences in treatment between the Hispanic and NHW population, as we saw no difference in the number of patients being treated and no difference in time to treatment $(P=0.3$, median 5 days for non-Hispanic, 3 days for Hispanic). However, our data suggests that this disparity was largely eliminated in the post-TKI era (Bencomo-Alvarez et al. 2020). Whereas being younger at diagnosis is considered a good prognostic factor for adult blood cancer patients, there are important therapeutic implications. In the case of $\mathrm{CML}$, younger patients were reported to exhibit a more aggressive disease (Pemmaraju et al. 2012). Therefore, more robust treatment strategies with second-generation TKIs like nilotinib or dasatinib may be a better course of front-line therapy for this high-risk patient population.

\section{FACTORS THAT CONTRIBUTE TO HEALTH DISPARITIES}

Cancer disparities in U.S. minority groups have been strongly associated with several potential risk factors, including both biological and nonbiological factors. Biological characteristics involving race and ethnicity may play important roles in driving the biology behind these disparities (Shoag et al. 2020). It is possible that the genomic variants listed above might contribute to reduced therapeutic responses in the Hispanic population, as reported previously (Lynce et al. 2016; Alanee et al. 2017; Moriyama et al. 2017; McClain et al. 2018). A combination of genomic mutations and differences in drug metabolism may also contribute to these disparities (ACS 2018). However, there are major challenges in identifying the causes of these cancer health disparities, as $<1 \%$ of biorepository specimens and only $2 \%$ of GWASs across the United States are from Hispanic individuals (Simon et al. 2014; Heredia et al. 2017). This greatly limits the identification of biomarkers or mutations in Hispanic cancer patients, thereby reducing the prospect of a personalized medicine approach in minorities. To address this issue, we have established a biorepository of primarily Mexican Hispanic blood cancer specimens from patients residing near the U.S./Mexican border. Our data and that of others suggest that not only Hispanic ethnicity, but also border proximity, associates with poor outcomes for certain types of cancers (Bencomo-Alvarez et al. 2020; Paulozzi et al. 2020). For patients living along the U.S./Mexican border regions spanning from California, Arizona, New Mexico, and Texas, Paulozzi et al. (2020) recently reported that Hispanics demonstrated higher mortality rates than non-Hispanic patients of $<35 \mathrm{yr}$ for multiple cancer sites (including leukemia). Additionally, they found that Hispanics are less likely than nonHispanics to be diagnosed at early stages of cancer and have lower survival rates. Previous research has suggested that the low death rates in cancer, heart, circulatory, and respiratory diseases in the Hispanic population could be explained by reduced smoking behaviors compared with the NHW population (Philips et al. 2013; McDonald and Paulozzi 2019). However, this assertion may be biased, because the main causes of mortality in $\mathrm{NHWs}$ are heart and circulatory system diseases, as well as lung cancer (ACS 2018). Furthermore, the highest cancer mortality rates in Hispanics occur in younger patients, so although smoking behaviors may have a negative impact on the overall mortality of many diseases, it is unlikely that we will see the harmful effect on this age group.

Nonbiological factors are also important contributors to cancer health disparities and likely play an even bigger role than biological traits. Nonbiological factors include low SES, residence in rural areas, and lack of access to health care in medically underserved regions like the U.S./Mexican border. Additional nonbiological factors include lower rates of 
cancer screening, which avoids a timely diagnosis and delays treatment (Shokar et al. 2019; Lee et al. 2020), as well as lower treatment adherence and distrust in the health-care system (Zavala et al. 2021). Language and literacy barriers also play a role. To overcome this, it is critical that clinical trials offer consent forms and information materials in both English and Spanish, to improve participation by certain minority groups. Additionally, occupational and environmental hazards may increase exposure of certain individuals to chemicals or radiation (Appelbaum 2020; Ramirez AG 2020). This may be true in the case of El Paso, as both Hispanic and NHW patients with ALL have worse outcomes than patients in other areas of Texas, implicating a possible environmental exposure. A summary of factors that influence cancer health disparities in minorities may be found in Figure 1.

In addition to the factors listed above, lack of insurance is a major problem in minority groups. Recent data shows that $72 \%$ of the Hispanic population in the United States has no health insurance, compared with just 10\% of the remaining population (Krakora et al. 2020). A lack of private health insurance influences access to critical therapies like allogeneic hematopoietic stem cell transplantation (allo-HSCT), one of the only curative treatment approaches available for blood cancer patients. Because allo-HSCT is a costly therapy, and government programs do not cover these procedures, a lack of health insurance makes it impossible to receive care in low-income populations. This inability to access critical therapies is reflected in higher mortality rates for minority groups (Joshua et al. 2010; Pulte et al. 2013; Thao et al. 2014; Jabo et al. 2017). Furthermore, minorities with low SES who do receive alloHSCT have worse outcomes (Baker et al. 2009). Hispanic blood cancer patients may also not have access to related or unrelated donors for allo-HSCT.

Reduced clinical trial enrollment and reduced access to treatment at comprehensive cancer centers across the United States also contribute to these cancer health disparities (Krakora

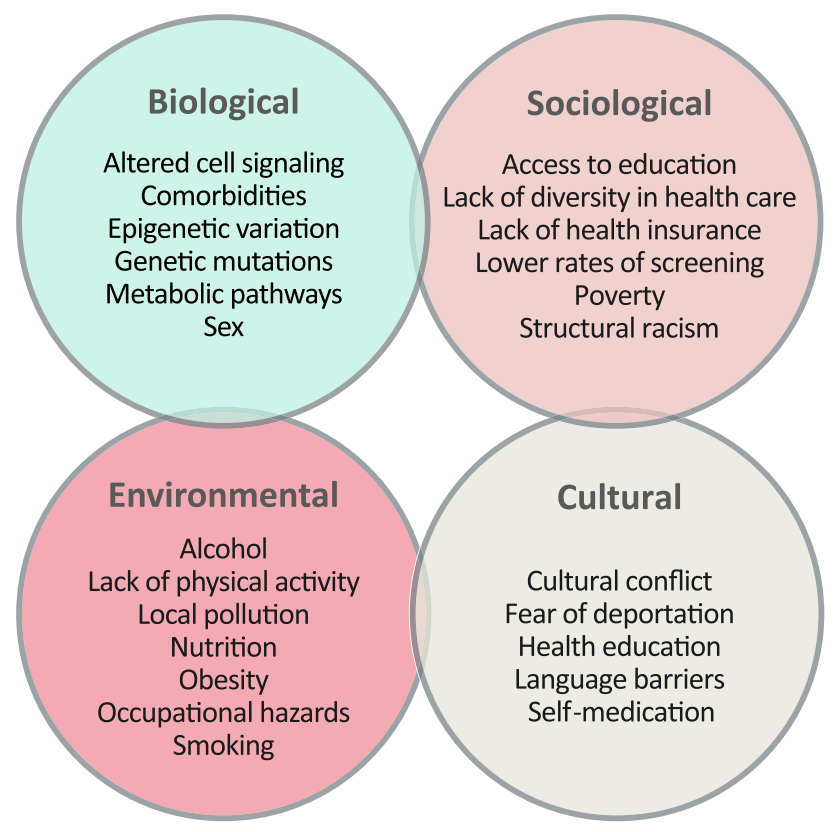

Figure 1. Factors contributing to cancer health disparities in minorities. The origin of cancer health disparities is caused by a complex interaction between biological, sociological, environmental, and cultural factors. These factors play important roles that contribute at different levels to the health status of patients, including access to health-care systems, the possibility of a timely diagnosis, and access to treatment options. A combination of these factors results in a higher cancer burden and higher incidence and mortality rates in certain minority groups. 
et al. 2020). Our own observations suggest that residence near the U.S./Mexican border is associated with worse outcomes; thus, regional location also plays a role (Bencomo-Alvarez et al. 2020). We speculate that this may be due to the lack of specialized cancer centers at the U.S./Mexican border. For example, Wolfson et al. (2017) reported that patients who were not treated at a National Cancer Institute-Designated Comprehensive Cancer Center (NCICCC) experienced worse OS compared with those treated at a NCICCC, and minority cancer patients were less likely to receive treatment at a NCICCC. Although El Paso is considered an urban area, the closest NCICCC is more than 200 miles away (in New Mexico), and treatment is complicated by factors including insurance coverage and overnight stays. Indeed, when we compared the OS of ALL patients diagnosed in the Houston/Dallas area on the East side of Texas with El Paso on the West side of Texas, patients diagnosed near Houston/Dallas had improved 10-yr survival (38\%) compared with patients diagnosed in El Paso (28\%) (Bencomo-Alvarez et al. 2020). Poorly resourced centers may be less able to provide the same level of acute care as a NCICCC. Additionally, the presence of comorbidities, such as diabetes, obesity, or metabolic syndrome, may also contribute to worse outcomes for Hispanic blood cancer patients (Kirtane and Lee 2017). Interplay between barriers to health care, age at diagnosis, and the presence of comorbidities place Hispanics at a risk for poor prognosis, especially for patients living at the U.S./Mexican border.

With regard to the "Hispanic paradox," which outlines reduced mortality rates for Hispanic cancer patients compared with non-Hispanics in the United States, despite having lower SES (McDonald and Paulozzi 2019), multiple groups have shown that Hispanics have lower mortality rates in cancer and other diseases (Sorlie et al. 1995; McDonald and Paulozzi 2019). These findings led to a theory that Hispanics had lower mortality rates due to the "healthy migrant" effect, suggesting that the majority of Hispanics who are in the United States are inherently healthier (Sorlie et al. 1995). Later, Palloni et al. found similar results, demonstrating that Hispanics have mortality advantages that were attributed not only to "healthy migrants," but also "salmon bias," suggesting that unhealthy migrants return to their home countries and die there (Palloni and Arias 2004). Another more recent theory argues that reduced rates of smoking in Hispanics explains the reduced cancer mortality rates (Blue and Fenelon 2011). Despite the "Hispanic paradox," Hispanic patients with certain types of cancers, especially acute leukemia, have higher incidence rates and worse overall survival.

One commonality between the four leukemias is that the Hispanic population tends to be diagnosed at a younger age compared with non-Hispanics (Hallek et al. 2010; Patel et al. 2012, 2013, 2015; Pulte et al. 2013; Nabhan et al. 2014; Kahn et al. 2016b; Jain et al. 2017; Sarmad et al. 2017; Krakora et al. 2020; Shoag et al. 2020), and we have confirmed these findings in a population residing near the U.S./Mexican border (BencomoAlvarez et al. 2020). The Hispanic population in the United States is generally younger than the non-Hispanic population (Schaeffer 2019); therefore, mortality rates from cancer or other diseases associated with advanced ages are lower in Hispanics. Although a younger age at diagnosis is often a good prognostic factor, it does not correlate with superior outcomes, especially for ALL and AML patients. The reasons for this observation are unknown and may simply reflect age differences in the population that have nothing to do with cancer. Alternatively, they could be explained by the increased comorbidities present in minority populations, which might offer patients improved access to care.

\section{CONCLUSIONS AND FUTURE DIRECTIONS}

The concept of cancer health disparities was first studied at the end of the last century. The primary aim was to identify the cause(s) underlying those disparities, in order to address them therapeutically, and to improve survival and quality of life for minority populations. However, 
although we understand which diseases have worse outcomes in minority populations, the underlying causes of these disparities are still not well-understood, leading to many unanswered questions. Do biological factors associated with race and ethnicity contribute to a cancer diagnosis, or are they simply biomarkers? Why are minority groups diagnosed at younger ages and at later stages of disease? How can we improve minority access to health insurance and health care? How can we improve representation of these groups in clinical trials in which new cancer treatments are developed? With current scientific evidence, we can convincingly state that cancer health disparities occur as a result of a mixture of various biological, environmental, and sociocultural factors, as well as language barriers and even occupational hazards to which minority groups are exposed. Polymorphisms and genetic mutations in Hispanics were shown to predispose patients to the development of certain cancers. Therefore, addressing these cancer health disparities will require a combination of strategies aimed at educating the population, providing access to care, and allowing enrollment into clinical trials for the development of new therapeutics.

As outlined above, there is sufficient evidence demonstrating cancer health disparities in the Hispanic population for several different blood cancers, especially ALL and AML. Ideally, for each individual disease, we need to better understand the biological and nonbiological factors contributing to blood cancer outcomes. Understanding the biological factors contributing to outcomes could lead us toward novel molecular targets and better therapeutic options for the Hispanic population. In contrast, understanding the nonbiological factors could lead to social and behavioral interventions aimed at reducing or eliminating the disparities (Fig. 2). In acute leukemia, genomic changes, low SES, high poverty rates, and lack of health insurance were associated with worse prognoses, outcomes, and survival. In chronic leukemia, on the other hand, only a few biological factors have been identified, but the same differences in social factors were recorded. Qualitative research studies will be required to elucidate the reasons underlying disparities in the Hispanic population and along the U.S./Mexican border region. Research would include surveys, interviews, and health system guidance, using community groups and "promotores." Promotores are health-care workers or health advocates that are trained to provide health education and prevention information in a culturally appropriate manner. Promotores have been highly successful in educating Hispanic/Latino communities about cancer health disparities, primarily promoting cancer screening in breast, cervical, and colorectal cancers (Fernández et al. 2009; Scheel et al. 2015; Molokwu et al. 2016).

Altogether, a combination of biological and nonbiological factors contribute to cancer health disparities for Hispanic blood cancer patients in the United States. To improve health care for minority groups, it is essential to investigate and identify the disparities that are present in the population. Identification of the disparity should lead to biomarker discovery, the development of new treatment approaches, and ultimately clinical trials, with the goal to establish a personalized medicine approach for minority groups (Fig. 2). Future studies should establish what is causing cancer health disparities in the Hispanic population, in order to identify novel interventions aimed at increasing access to health care and improving survival and quality of life for the largest and fastest growing minority group in the United States.

\section{ADDITIONAL INFORMATION}

\section{Acknowledgments}

The authors thank Dr. Rajkumar Lakshmanaswamy for his supervision, mentorship, and support. We also acknowledge Drs. Attilio Orazi, Osvaldo Padilla, Alexander Philipovskiy, Sumit Gaur, and Javier Corral for their contributions toward establishing a biorepository of primarily Hispanic leukemia specimens at Texas Tech University Health Sciences Center at El Paso. 


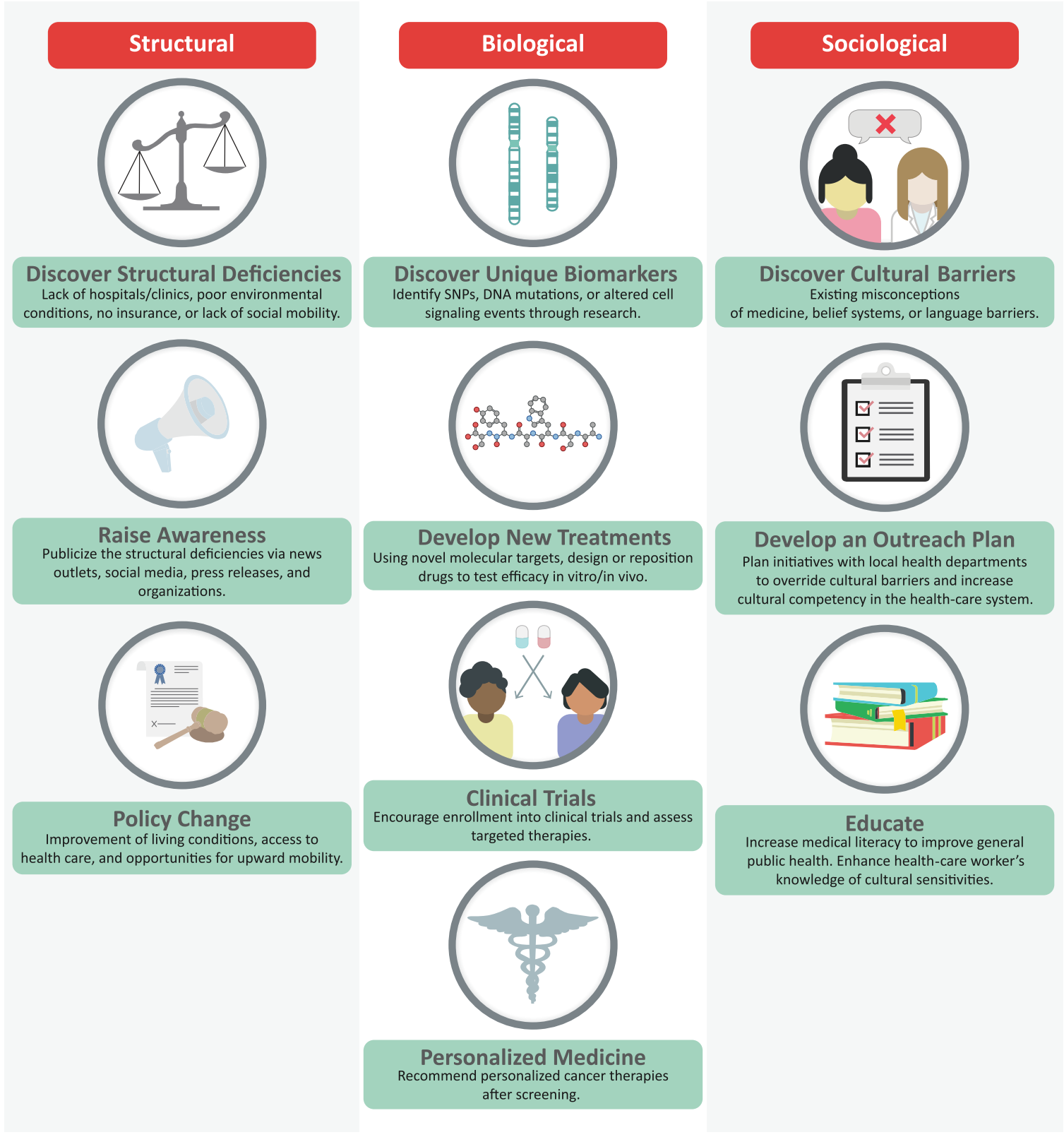

Figure 2. Improving health care for minority groups. To improve health care for minority groups, it is essential to first identify the disparities that are present in minorities. These disparities can be grouped into three categories: (1) structural (left), (2) biological (middle), and (3) sociological (right). Structural disparities manifest through the unequal demographic distribution of minorities into areas with low economic opportunity, reduced educational opportunities, and poor living conditions. To ameliorate structural deficiencies, it is essential to raise awareness of these issues so that policies that advocate for minorities could be made. Identification of biological disparities may lead to the discovery of biomarkers unique to understudied racial/ethnic groups, which in turn leads to the development of new treatment options and improved enrollment in clinical trials. The ultimate goal is to establish a personalized approach for care of minority blood cancer patients. Last, sociological factors manifest as impediments to health care in minorities, through cultural belief systems, erroneous misconception of medicine, or ignorance from health-care providers regarding cultural sensitivities. Overcoming these obstacles can be done by putting efforts into education, with the end goal of increasing medical literacy within minority groups, while implementing a more culturally competent health-care system. 
Competing Interest Statement

The authors have declared no competing interest.

\section{Funding}

A.M.E. acknowledges support from the National Cancer Institute of the National Institutes of Health under award number K22CA216008. The content is solely the responsibility of the authors and does not necessarily represent the official views of the National Institutes of Health.

\section{REFERENCES}

ACS. 2018. Cancer facts \& figures for Hispanics/Latinos 2018-2020. American Cancer Society, Atlanta. ACS. 2020. Cancer Facts \& Figures 2020. American Cancer Society, Atlanta.

Alanee SR, Shah S, Zabor EC, Vijai J, Ostrovnaya I, Garcia-Grossman IR, Pendse DV, Littman J, Regazzi AM, Offit $\mathrm{K}$, et al. 2017. Evaluating the association of multiple single nucleotide polymorphisms with response to gemcitabine and platinum combination chemotherapy in urothelial carcinoma of the bladder. Int J Clin Pharmacol Ther 55: 203-209. doi:10.5414/CP202856

Appelbaum FR. 2020. Acute leukemias in adults. In Abeloff's clinical oncology, 6th ed (ed. Niederhuber JE, Armitage JO, Kastan MB, et al.), pp. 1783-1797.e1781. Elsevier, Philadelphia.

Baker KS, Davies SM, Majhail NS, Hassebroek A, Klein JP, Ballen KK, Bigelow CL, Frangoul HA, Hardy CL, Bredeson C, et al. 2009. Race and socioeconomic status influence outcomes of unrelated donor hematopoietic cell transplantation. Biol Blood Marrow Transplant 15: 1543-1554. doi:10.1016/j.bbmt.2009.07 .023

Barrington-Trimis JL, Cockburn M, Metayer C, Gauderman WJ, Wiemels J, McKean-Cowdin R. 2015. Rising rates of acute lymphoblastic leukemia in Hispanic children: trends in incidence from 1992 to 2011. Blood 125: 3033-3034. doi:10.1182/blood-2015-03-634006

Bencomo-Alvarez AE, Gonzalez MA, Rubio AJ, Olivas IM, Lara JJ, Padilla O, Orazi A, Corral J, Philipovskiy A, Gaur S, et al. 2020. Ethnic and border differences on blood cancer presentation and outcomes: a Texas population-based study. Cancer doi:10.1002/cncr.33347.

Bhatnagar B, Kohlschmidt J, Mrozek K, Zhao Q, Fisher JL, Nicolet D, Walker CJ, Mims AS, Oakes C, Giacopelli $B$, et al. 2020. Poor survival and differential impact of genetic features of Black patients with acute myeloid leukemia. Cancer Discov. doi:10.1158/2159-8290.CD-20-1579.

Blue L, Fenelon A. 2011. Explaining low mortality among US immigrants relative to native-born Americans: the role of smoking. Int J Epidemiol 40: 786-793. doi:10.1093/ije/dyr011

Carrillo T, Montealegre JR, Bracamontes CG, Scheurer ME, Follen M, Mulla ZD. 2021. Predictors of timely diagnostic follow-up after an abnormal Pap test among Hispanic women seeking care in El Paso, Texas. BMC Womens Health 21: 11. doi:10.1186/s12905-020-01161-9

DaNavas-Walt C, Proctor BD. 2015. Income and poverty in the United States: 2014. U.S. Census Bureau, Washington, DC.

Darbinyan K, Shastri A, Budhathoki A, Helbig D, Snyder R, Pradhan K, Saleh-Esa J, Kornblum NS, Binder AF, Goel S, et al. 2017. Hispanic ethnicity is associated with younger age at presentation but worse survival in acute myeloid leukemia. Blood Adv 1: 2120-2123. doi:10.1182/bloodadvances.2017007013

de Heer HD, Balcazar HG, Morera OF, Lapeyrouse L, Heyman JM, Salinas J, Zambrana RE. 2013. Barriers to care and comorbidities along the U.S.-Mexico border. Public Health Rep 128: 480-488. doi:10.1177/ 003335491312800607

Deshpande A, Gramatges MM, Sasa GS, Lupo PJ, Rau RE, Redell MS, Horton TM, Scheurer ME, Rabin KR. 2015. Poorer relapse-free survival in Hispanic children diagnosed with acute myeloid leukemia compared with non-Hispanics: a Texas single institution experience. Blood 126: 1312. doi:10.1182/blood.V126.23 .1312 .1312

Di Bernardo MC, Crowther-Swanepoel D, Broderick P, Webb E, Sellick G, Wild R, Sullivan K, Vijayakrishnan J, Wang Y, Pittman AM, et al. 2008. A genome-wide association study identifies six susceptibility loci for chronic lymphocytic leukemia. Nat Genet 40: 1204-1210. doi:10.1038/ng.219

Fajardo-Gutiérrez A, Juárez-Ocaña S, González-Miranda G, Palma-Padilla V, Carreón-Cruz R, Ortega-Alvárez MC, Mejía-Arangure JM. 2007. Incidence of cancer in children residing in ten jurisdictions of the Mexican Republic: importance of the Cancer registry (a population-based study). BMC Cancer 7: 68. doi:10.1186/ 1471-2407-7-68

Fernández ME, Gonzales A, Tortolero-Luna G, Williams J, Saavedra-Embesi M, Chan W, Vernon SW. 2009. Effectiveness of Cultivando la Salud: a breast and cervical cancer screening promotion program for low-income Hispanic women. Am J Public Health 99: 936-943. doi:10.2105/AJPH.2008.136713

Florea A, Brown HE, Harris RB, Oren E. 2019. Ethnic disparities in gastric cancer presentation and screening practice in the United States: analysis of 1997-2010 surveillance, epidemiology, and end 
results-Medicare data. Cancer Epidemiol Biomarkers Prev 28: 659-665. doi:10.1158/1055-9965.EPI-180471

Gomez-Arteaga A, Mencia-Trinchant N, Tovar AM, Carrillo IO, Laguna AG, Fuller ER, Penafiel CR, Guzman ML, Hassane DC. 2020. Abstract C061: molecular landscape of acute myeloid leukemia in Mexico. Cancer Epidemiol Biomarkers Prev 29: C061.

Gramatges MM, Deshpande A, Lupo PJ, Rau RE, Redell ML, Horton TM, Scheurer ME, Rabin KR. 2017. Ethnic disparities relative to disease features and outcomes in children with acute myeloid leukemia. Pediatr Blood Cancer 64. doi:10.1002/pbc.26487

Gurdasani D, Barroso I, Zeggini E, Sandhu MS. 2019. Genomics of disease risk in globally diverse populations. Nat Rev Genet 20: 520-535. doi:10.1038/s41576-019-0144-0

Hallek M. 2019. Chronic lymphocytic leukemia: 2020 update on diagnosis, risk stratification and treatment. Am J Hematol 94: 1266-1287. doi:10.1002/ajh.25595

Hallek M, Fischer K, Fingerle-Rowson G, Fink AM, Busch R, Mayer J, Hensel M, Hopfinger G, Hess G, von Grünhagen U, et al. 2010. Addition of rituximab to fludarabine and cyclophosphamide in patients with chronic lymphocytic leukaemia: a randomised, open-label, phase 3 trial. Lancet 376: 1164-1174. doi:10 .1016/S0140-6736(10)61381-5

Heredia NI, Krasny S, Strong LL, Von Hatten L, Nguyen L, Reininger BM, McNeill LH, Fernandez ME. 2017. Community perceptions of biobanking participation: a qualitative study among Mexican-Americans in three Texas cities. Public Health Genomics 20: 46-57. doi:10.1159/000452093

Hernandez-Caballero A, Arellano-Llamas AA, Cruz-Rico J, Ojeda JV, Tuna-Aguilar E, Aguayo-Gonzalez A, Oropeza-Martinez MP, Montiel-Cervantes LA, Anaya LS, Canizales-Quinteros S, et al. 2015. Genetic susceptibility variants for chronic lymphocytic leukaemia in Mexican mestizos. Br J Haematol 169: 909-911. doi:10.1111/bjh.13259

Hindorff LA, Bonham VL, Brody LC, Ginoza MEC, Hutter CM, Manolio TA, Green ED. 2018. Prioritizing diversity in human genomics research. Nat Rev Genet 19: 175-185. doi:10.1038/nrg.2017.89

Höglund M, Sandin F, Simonsson B. 2015. Epidemiology of chronic myeloid leukaemia: an update. Ann Hematol 94: 241-247. doi:10.1007/s00277-015-2314-2

Jabbour E, Kantarjian H. 2020. Chronic myeloid leukemia: 2020 update on diagnosis, therapy and monitoring. Am J Hematol 95: 691-709. doi:10.1002/ajh.25792

Jabo B, Morgan JW, Martinez ME, Ghamsary M, Wieduwilt MJ. 2017. Sociodemographic disparities in chemotherapy and hematopoietic cell transplantation utilization among adult acute lymphoblastic and acute myeloid leukemia patients. PLoS One 12: e0174760. doi:10.1371/journal.pone.0174760

Jain N, Roberts KG, Jabbour E, Patel K, Eterovic AK, Chen K, Zweidler-McKay P, Lu X, Fawcett G, Wang SA, et al. 2017. Ph-like acute lymphoblastic leukemia: a high-risk subtype in adults. Blood 129: 572-581. doi:10 .1182/blood-2016-07-726588

Joshua TV, Rizzo JD, Zhang MJ, Hari PN, Kurian S, Pasquini M, Majhail NS, Lee SJ, Horowitz MM. 2010. Access to hematopoietic stem cell transplantation: effect of race and sex. Cancer 116: 3469-3476. doi:10.1002/ cncr.25297

Julio D, Ferran N, Dolors C, Elias C. 2020. Chronic lymphocytic leukemia: from molecular pathogenesis to novel therapeutic strategies. Haematologica 105: 2205-2217. doi:10.3324/haematol.2019.236000

Kahn JM, Keegan TH, Tao L, Abrahão R, Bleyer A, Viny AD. 2016a. Racial disparities in the survival of American children, adolescents, and young adults with acute lymphoblastic leukemia, acute myelogenous leukemia, and Hodgkin lymphoma. Cancer 122: 2723-2730. doi:10.1002/cncr.30089

Kahn JM, Keegan THM, Tao L, Abrahão R, Bleyer A, Viny AD. 2016b. Racial disparities in the survival of American children, adolescents, and young adults with acute lymphoblastic leukemia, acute myelogenous leukemia, and Hodgkin lymphoma. Cancer 122: 2723-2730. doi:10.1002/cncr.30089

Kirtane K, Lee SJ. 2017. Racial and ethnic disparities in hematologic malignancies. Blood 130: 1699-1705. doi:10.1182/blood-2017-04-778225

Krakora R, Shih W, Popli P, Gorshein E, Salaru G, Moore D, Chen C, David K, Bannerji R. 2020. Impact of insurance status on survival outcomes in adults with acute lymphoblastic leukemia (ALL): a single-center experience. Clin Lymphoma Myeloma Leukemia 20: e890. doi:10.1016/j.clml.2020.06.010

Lee JP, Birnstein E, Masiello D, Yang D, Yang AS. 2009. Gender and ethnic differences in chronic myelogenous leukemia prognosis and treatment response: a single-institution retrospective study. $J$ Hematol Oncol 2: 30. doi:10.1186/1756-8722-2-30

Lee D-C, Liang H, Chen N, Shi L, Liu Y. 2020. Cancer screening among racial/ethnic groups in health centers. Int J Equity Health 19: 43. doi:10.1186/s12939-020-1153-5

Lim JY, Bhatia S, Robison LL, Yang JJ. 2014. Genomics of racial and ethnic disparities in childhood acute lymphoblastic leukemia. Cancer 120: 955-962. doi:10.1002/cncr.28531

Lynce F, Graves KD, Jandorf L, Ricker C, Castro E, Moreno L, Augusto B, Fejerman L, Vadaparampil ST. 2016. Genomic disparities in breast cancer among Latinas. Cancer Control 23: 359-372. doi:10.1177/ 107327481602300407 
McClain CA, Bernhardt MB, Berger A, Bernini JC, Marquez-Do D, Winslow R, Scheurer ME, Schafer ES. 2018. Pharmacogenetic association with neurotoxicity in Hispanic children with acute lymphoblastic leukaemia. Br J Haematol 181: 684-687. doi:10.1111/bjh.14678

McDonald JA, Paulozzi LJ. 2019. Parsing the paradox: Hispanic mortality in the US by detailed cause of death. J Immigr Minor Health 21: 237-245. doi:10.1007/s10903-018-0737-2

Minciacchi VR, Kumar R, Krause DS. 2021. Chronic myeloid leukemia: a model disease of the past, present and future. Cells 10: 117. doi:10.3390/cells10010117

Molina Garay C, Carrillo Sanchez K, Flores Lagunes LL, Jimenez Olivares M, Munoz Rivas A, Villegas Torres BE, Flores Aguilar H, Nunez Enriquez JC, Jimenez Hernandez E, Bekker Mendez VC, et al. 2020. Profiling FLT3 mutations in Mexican acute myeloid leukemia pediatric patients: impact on overall survival. Front Pediatr 8: 586. doi:10.3389/fped.2020.00586

Molokwu J, Penaranda E, Flores S, Shokar NK. 2016. Evaluation of the effect of a promotora-led educational intervention on cervical cancer and human papillomavirus knowledge among predominantly Hispanic primary care patients on the US-Mexico border. J Cancer Educ 31: 742-748. doi:10.1007/s13187-0150938-5

Moriyama T, Yang YL, Nishii R, Ariffin H, Liu C, Lin TN, Yang W, Lin DT, Yu CH, Kham S, et al. 2017. Novel variants in NUDT15 and thiopurine intolerance in children with acute lymphoblastic leukemia from diverse ancestry. Blood 130: 1209-1212. doi:10.1182/blood-2017-05-782383

Mughal TI, Radich JP, Deininger MW, Apperley JF, Hughes TP, Harrison CJ, Gambacorti-Passerini C, Saglio G, Cortes J, Daley GQ. 2016. Chronic myeloid leukemia: reminiscences and dreams. Haematologica 101: 541-558. doi:10.3324/haematol.2015.139337

NAACCR. 2011. NAACCR Race and Ethnicity Work Group. NAACCR Guideline for Enhancing Hispanic/Latino Identification: Revised NAACCR Hispanic/Latino Identification Algorithm [NHIA v2.2.1]. North American Association of Central Cancer Registries, Springfield, IL.

Nabhan C, Aschebrook-Kilfoy B, Chiu BCH, Smith SM, Shanafelt TD, Evens AM, Kay NE. 2014. The impact of race, ethnicity, age and sex on clinical outcome in chronic lymphocytic leukemia: a comprehensive surveillance, epidemiology, and end results analysis in the modern era. Leuk Lymphoma 55: 2778-2784. doi:10 $.3109 / 10428194.2014 .898758$

National Academies of Sciences, Engineering, and Medicine; Health and Medicine Division; Board on Population Health and Public Health Practice; Committee on Community-Based Solutions to Promote Health Equity in the United, States. 2017. The state of health disparities in the United States, Ch. 2, pp. 57-88. In Communities in action: pathways to health equity (ed. Baciu A, Negussie Y, Geller A, et al.). National Academies Press, Washington, DC.

O'Hare T, Zabriskie MS, Eiring AM, Deininger MW. 2012. Pushing the limits of targeted therapy in chronic myeloid leukaemia. Nat Rev Cancer 12: 513-526. doi:10.1038/nrc3317

Palloni A, Arias E. 2004. Paradox lost: explaining the Hispanic adult mortality advantage. Demography 41: 385-415. doi:10.1353/dem.2004.0024

Patel MI, Ma Y, Mitchell BS, Rhoads KF. 2012. Understanding disparities in leukemia: a national study. Cancer Causes Control 23: 1831-1837. doi:10.1007/s10552-012-0062-3

Patel MI, Ma Y, Mitchell BS, Rhoads K. 2013. Are racial and ethnic disparities in acute myeloid leukemia due to differences in treatment? Blood 122: 726. doi:10.1182/blood.V122.21.726.726

Patel MI, Ma Y, Mitchell BS, Rhoads KF. 2015. Age and genetics: how do prognostic factors at diagnosis explain disparities in acute myeloid leukemia? Am J Clin Oncol 38: 159-164. doi:10.1097/COC $.0 \mathrm{~b} 013 \mathrm{e} 31828 \mathrm{~d} 7536$

Patel MI, Lopez AM, Blackstock W, Reeder-Hayes K, Moushey A, Phillips J, Tap W. 2020. Cancer disparities and health equity: a policy statement from the American Society of Clinical Oncology. J Clin Oncol 38: 34393448. doi:10.1200/JCO.20.00642

Paulozzi LJ, McDonald JA, Sroka CJ. 2020. A disparity beneath a paradox: cancer mortality among young Hispanic Americans in the US-Mexico border region. J Racial Ethn Health Disparities doi:10.1007/ s40615-020-00920-6

Pemmaraju N, Kantarjian H, Shan J, Jabbour E, Quintas-Cardama A, Verstovsek S, Ravandi F, Wierda W, O'Brien S, Cortes J. 2012. Analysis of outcomes in adolescents and young adults with chronic myelogenous leukemia treated with upfront tyrosine kinase inhibitor therapy. Haematologica 97: 1029-1035. doi:10 .3324/haematol.2011.056721

Perkins K, Wiley S. 2014. Minorities. In Encyclopedia of critical psychology (ed. Teo T), pp. 1192-1195. Springer New York.

Philips BU Jr, Belasco E, Markides KS, Gong G. 2013. Socioeconomic deprivation as a determinant of cancer mortality and the Hispanic paradox in Texas, USA. Int J Equity Health 12: 26. doi:10.1186/1475-9276-12-26

Pollyea D, Kohrt H, Yang J, Chang E, Gomez S, Clarke C. 2014. Acute leukemia in adult Hispanic Americans: differences in incidence rates by nativity. J Cancer Prev Curr Res 1: 00005. doi:10.15406/jcpcr.2014.01 .00005 
Pulte D, Redaniel MT, Jansen L, Brenner H, Jeffreys M. 2013. Recent trends in survival of adult patients with acute leukemia: overall improvements, but persistent and partly increasing disparity in survival of patients from minority groups. Haematologica 98: 222-229. doi:10.3324/haematol.2012.063602

Rajabi B, Corral JC, Hakim N, Mulla ZD. 2012. Descriptive epidemiology of gastric adenocarcinoma in the state of Texas by ethnicity: Hispanic versus White non-Hispanic. Gastric Cancer 15: 405-413. doi:10.1007/ s10120-011-0127-0

Ramirez AG TE. 2020. Advancing the science of cancer in Latinos. Springer, New York.

Rebbeck TR, Burns-White K, Chan AT, Emmons K, Freedman M, Hunter DJ, Kraft P, Laden F, Mucci L, Parmigiani G, et al. 2018. Precision prevention and early detection of cancer: fundamental principles. Cancer Discov 8: 803-811. doi:10.1158/2159-8290.CD-17-1415

Sarmad R, Jamy O, Costa LJ. 2017. Survival of chronic myeloid leukemia patients in comparison to general population in the tyrosine kinase inhibitors era: a US population based study. Blood 130: 2165.

Schaeffer K. 2019. The most common age among whites in U.S. is 58-more than double that of racial and ethnic minorities. Pew Research Center. https://www.pewresearch.org/fact-tank/2019/07/30/mostcommon-age-among-us-racial-ethnic-groups/

Scheel JR, Molina Y, Briant KJ, Ibarra G, Lehman CD, Thompson B. 2015. Latinas' mammography intention following a home-based promotores-led intervention. J Commun Health 40: 1185-1192. doi:10.1007/ s10900-015-0046-9

SEER. 2020. Cancer state facts: leukemia. National Cancer Institute, Bethesda, MD.

Shoag JM, Barredo JC, Lossos IS, Pinheiro PS. 2020. Acute lymphoblastic leukemia mortality in Hispanic Americans. Leuk Lymphoma 61: 2674-2681. doi:10.1080/10428194.2020.1779260

Shokar NK, Calderon-Mora J, Molokwu J, Byrd T, Alomari A, Mallawaarachchi I, Dwivedi A. 2019. Outcomes of a multicomponent culturally tailored cervical cancer screening intervention among underserved Hispanic women (De Casa en Casa). Health Promot Pract 22: 112-121. doi:10.1177/1524839919893309

Siegel DA, Henley SJ, Li J, Pollack LA, Van Dyne EA, White A. 2017. Rates and trends of pediatric acute lymphoblastic leukemia-United States, 2001-2014. MMWR Morb Mortal Wkly Rep 66: 950-954. doi:10 .15585/mmwr.mm6636a3

Siegel RL, Miller KD, Fuchs HE, Jemal A. 2021. Cancer statistics, 2021. CA Cancer J Clin 71: 7-33. doi:10.3322/ caac. 21654

Simon MA, de la Riva EE, Bergan R, Norbeck C, McKoy JM, Kulesza P, Dong X, Schink J, Fleisher L. 2014. Improving diversity in cancer research trials: the story of the cancer disparities research network. $J$ Cancer Educ 29: 366-374. doi:10.1007/s13187-014-0617-y

Slager SL, Goldin LR, Strom SS, Lanasa MC, Spector LG, Rassenti L, Leis JF, Camp NJ, Kay NE, Vachon CM, et al. 2010. Genetic susceptibility variants for chronic lymphocytic leukemia. Cancer Epidemiol Biomarkers Prev 19: 1098-1102. doi:10.1158/1055-9965.EPI-09-1217

Sorlie PD, Backlund E, Keller JB. 1995. US mortality by economic, demographic, and social characteristics: the national longitudinal mortality study. Am J Public Health 85: 949-956. doi:10.2105/AJPH.85.7.949

Springfield SA, Van Duyn MAS, Aguila HN, Ogunbiyi P, Bailey LO, Lin A, Wallace TA, Snyder FR. 2020. The NCl center to reduce cancer health disparities: moving forward to eliminate cancer health disparities and diversify the cancer biomedical workforce. J Natl Med Assoc 112: 308-314. doi:10.1016/j.jnma.2020.06 .001

Stauder R, Lambert J, Desruol-Allardin S, Savre I, Gaugler L, Stojkov I, Siebert U, Chevrou-Séverac H. 2020. Patient-reported outcome measures in studies of myelodysplastic syndromes and acute myeloid leukemia: literature review and landscape analysis. Eur J Haematol 104: 476-487. doi:10.1111/ejh.13389

Swords R, Sznol J, Elias R, Watts J, Zelent A, Martin E, Vargas F, Bethel-Ellison S, Kobetz E. 2016. Acute leukemia in adult Hispanic Americans: a large-population study. Blood Cancer J 6: e484. doi:10.1038/bcj .2016 .94

Thao V, Kozhimannil KB, Thomas W, Golberstein E. 2014. Variation in inpatient costs of hematopoietic cell transplantation among transplant centers in the United States. JAMA Int Med 174: 1409-1412. doi:10 .1001/jamainternmed.2014.2302

Turner A. 2018. The business case for racial equity a strategy for growth. WK Kellogg Foundation and Altarum. https://altarum.org/RacialEquity2018

Utuama O, Mukhtar F, Pham YT, Dabo B, Manani P, Moser J, Michael-Asalu A, Tran CT, Le LC, Le TV, et al. 2019. Racial/ethnic, age and sex disparities in leukemia survival among adults in the United States during 1973-2014 period. PLoS One 14: e0220864. doi:10.1371/journal.pone.0220864

Wang L, Bhatia S, Gomez SL, Yasui Y. 2015. Differential inequality trends over time in survival among U.S. children with acute lymphoblastic leukemia by race/ethnicity, age at diagnosis, and sex. Cancer Epidemiol Biomarkers Prev 24: 1781-1788. doi:10.1158/1055-9965.EPI-15-0639

Wolfson J, Sun CL, Wyatt L, Stock W, Bhatia S. 2017. Adolescents and young adults with acute lymphoblastic leukemia and acute myeloid leukemia: impact of care at specialized cancer centers on survival outcome. Cancer Epidemiol Biomarkers Prev 26: 312-320. doi:10.1158/1055-9965.EPI-16-0722 
Yanez B, McGinty HL, Buitrago D, Ramirez AG, Penedo FJ. 2016. Cancer outcomes in Hispanics/Latinos in the United States: an integrative review and conceptual model of determinants of health. J Lat Psychol 4: 114129. doi:10.1037/lat0000055

Zavala VA, Bracci PM, Carethers JM, Carvajal-Carmona L, Coggins NB, Cruz-Correa MR, Davis M, de Smith AJ, Dutil J, Figueiredo JC, et al. 2021. Cancer health disparities in racial/ethnic minorities in the United States. Br J Cancer 124: 315-332. doi:10.1038/s41416-020-01038-6

Zhao Y, Wang Y, Ma S. 2018. Racial differences in four leukemia subtypes: comprehensive descriptive epidemiology. Sci Rep 8: 548. doi:10.1038/s41598-017-19081-4 


\section{COLD SPRING HARBOR Molecular Case Studies}

\section{Blood cancer health disparities in the United States Hispanic population}

Alfonso E. Bencomo-Alvarez, Andres J. Rubio, Mayra A. Gonzalez, et al.

Cold Spring Harb Mol Case Stud 2021, 7: a005967 originally published online February 16, 2021 Access the most recent version at doi: $10.1101 / \mathrm{mcs} .0005967$

References This article cites 76 articles, 16 of which can be accessed free at: http://molecularcasestudies.cshlp.org/content/7/2/a005967.full.html\#ref-list-1

License This article is distributed under the terms of the Creative Commons Attribution-NonCommercial License, which permits reuse and redistribution, except for commercial purposes, provided that the original author and source are credited.

Email Alerting Receive free email alerts when new articles cite this article - sign up in the box at the Service top right corner of the article or click here. 